\title{
Single-Spin Asymmetries and Transversity
}

\author{
Philip G. Ratcliffe \\ Dip.to di Scienze CC.FF.MM., Univ. degli Studi dell'Insubria—sede di Como, Italy \\ and INFN—sezione di Milano, Italy
}

\begin{abstract}
A pedagogical introduction to single-spin asymmetries (SSA's) and transversity is presented. Discussion in some detail is made of certain aspects of SSA's in lepton-nucleon and in hadron-hadron scattering and the role of $\mathrm{pQCD}$ and evolution in the context of transversity.
\end{abstract}

\section{PREAMBLE}

Single-spin asymmetries are one of the oldest forms of high-energy spin measurement, the reason being accessibility: the only requirement is either beam or target polarised, for $\Lambda^{0}$ production neither is necessary! However, after early interest (due to large experimental effects), a theoretical "dark age" descended: pQCD had apparently nothing to say, save that such asymmetries are zero! We now know that the rich phenomenology is matched by a richness of the theoretical framework: the main topic of my talk.

One might argue the inapplicability of pQCD to existing SSA data owing to the low $Q^{2}$ accessed while there are several non-pQCD models that can explain some (though not all) of the data. Examples may be found in [1, 2, 3, 4]. However, I shall examine SSA's purely from within the $\mathrm{pQCD}$ framework.

Transversity too has a long history: the concept (though not the term) was introduced in 1979 by Ralston and Soper via the Drell-Yan process. The leading order (LO) anomalous dimensions were first calculated by Baldracchini et al. [6] but forgotten. They were recalculated by Artru and Mekhfi [7] and it turns out that they had also been obtained by various groups as part of the $g_{2}$ evolution [8, 9, 10, 11]. A complete classification of chirally-odd densities including transversity, is due to Jaffe and Ji [12]. However, as yet there are no experimental data on transversity. This is owing to the inaccessibility (discussed later) of transversity in inclusive deeply-inelastic scattering (DIS).

After introducing single-spin asymmetries and transversity, I shall discuss SSA's in lepton-nucleon and hadron-hadron scattering in some detail and close with a few brief comments and conclusions. A large part of what follows is taken from the Physics Reports by Barone, Drago and Ratcliffe [13] and from a forthcoming book by Barone and Ratcliffe [14]. Thus, much credit is due to my two collaborators.

\section{INTRODUCTION}

Generically, SSA's reflect correlations of the form $\mathbf{s} \cdot(\mathbf{p} \wedge \mathbf{k})$, where $\mathbf{s}$ is a polarisation vector while $\mathbf{p}$ and $\mathbf{k}$ are particle/jet momenta. An example is $\mathbf{s}$ a (transverse) target 
polarisation, $\mathbf{p}$ a beam direction, and $\mathbf{k}$ that of a final-state particle. Thus, polarisations in SSA's will typically be transverse (but see later). Transforming the basis from transverse spin to the more familiar helicity, $|\uparrow / \downarrow\rangle=\frac{1}{\sqrt{ } 2}[|+\rangle \pm \mathrm{i}|-\rangle]$, such an asymmetry takes on the (schematic) form

$$
A \sim \frac{\langle\uparrow \mid \uparrow\rangle-\langle\downarrow \mid \downarrow\rangle}{\langle\uparrow \mid \uparrow\rangle+\langle\downarrow \mid \downarrow\rangle} \sim \frac{2 \operatorname{Im}\langle+\mid-\rangle}{\langle+\mid+\rangle+\langle-\mid-\rangle} .
$$

The presence of both $|+\rangle$ and $|-\rangle$ in the numerator implies a spin-flip amplitude while the precise form indicates interference between spin-flip and non-flip amplitudes, with a non-trivial relative phase difference.

It was soon realised [15] that in the Born approximation and massless limit a gauge theory, such as quantum chromodynamics (QCD), cannot furnish either requirement since fermion helicity is conserved and tree diagrams are real. Quoting from [15]: "... observation of significant polarizations in the above reactions would contradict either QCD or its applicability." Later, however, examining the three-parton correlators related to $g_{2}$, Efremov and Teryaev [16] found a way out: the mass scale relevant to spin flip is not that of a current quark but the hadron and the two-loop nature of the diagrams can give rise to an imaginary part. Nonetheless, it was a while before the complexity of the new structures was fully exploited (see, e.g., [17, 18]).

Transversity is the third twist-two partonic density. At this point it is important to make the distinction between partonic densities $-q(x), \Delta q(x), \Delta_{T} q(x), \ldots$ and DIS structure functions $-F_{1}, F_{2}, g_{1}, g_{2}, \ldots$ In the leading-twist unpolarised and helicitydependent cases there is a simple connection between the two: DIS structure functions are weighted sums of partonic densities; in contrast, there is no DIS transversity structure function and $g_{2}$ does not correspond to a partonic density. The absence of transversity in DIS is illustrated in Fig. 1. Note that chirality flip is not a problem if the quarks connect

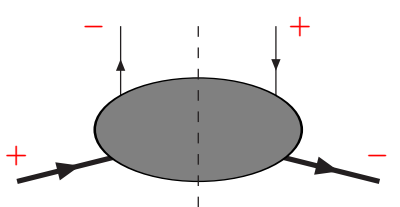

(a)

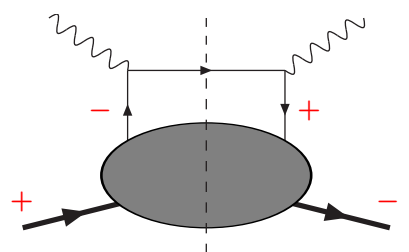

(b)

FIGURE 1. (a) Chirally-odd hadron-quark amplitude, (b) chirality-flip forbidden DIS diagram.

to different hadrons, as in Drell-Yan processes.

The three twist-two structures are then:

$$
\begin{aligned}
f(x) & =\int \frac{\mathrm{d} \xi^{-}}{4 \pi} \mathrm{e}^{\mathrm{i} x P^{+} \xi^{-}}\left\langle P S\left|\bar{\psi}(0) \gamma^{+} \psi\left(0, \xi^{-}, \mathbf{0}_{\perp}\right)\right| P S\right\rangle \\
\Delta f(x) & =\int \frac{\mathrm{d} \xi^{-}}{4 \pi} \mathrm{e}^{\mathrm{i} x P^{+} \xi^{-}}\left\langle P S\left|\bar{\psi}(0) \gamma^{+} \gamma_{5} \psi\left(0, \xi^{-}, \mathbf{0}_{\perp}\right)\right| P S\right\rangle, \\
\Delta_{T} f(x) & =\int \frac{\mathrm{d} \xi^{-}}{4 \pi} \mathrm{e}^{\mathrm{i} x P^{+} \xi^{-}}\left\langle P S\left|\bar{\psi}(0) \gamma^{+} \gamma^{1} \gamma_{5} \psi\left(0, \xi^{-}, \mathbf{0}_{\perp}\right)\right| P S\right\rangle .
\end{aligned}
$$

The $\gamma_{5}$ matrix signals spin dependence while the extra $\gamma^{1}$ matrix in $\Delta_{T} f(x)$ signals the chirality-flip that precludes transversity contributions in DIS. 
For somewhat similar reasons the LO QCD evolution of transversity is non-singlet like: quark-gluon mixing would require a chirality-flip in a quark propagator-see

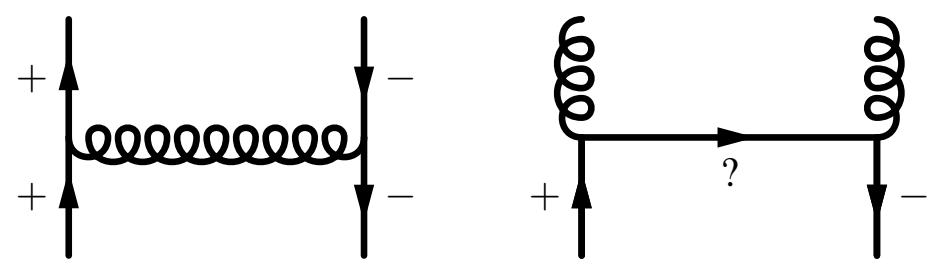

FIGURE 2. Left, transversity evolution kernel; right, disallowed gluon-fermion mixing.

Fig. ․ The LO quark-quark splitting functions are then:

$$
\begin{aligned}
P_{q q}^{(0)} & =C_{\mathrm{F}}\left(\frac{1+x^{2}}{1-x}\right)_{+}, \\
\Delta P_{q q}^{(0)} & =P_{q q}^{(0)} \quad(\text { by helicity conservation), } \\
\Delta_{T} P_{q q}^{(0)} & =C_{\mathrm{F}}\left[\left(\frac{1+x^{2}}{1-x}\right)_{+}-1+x\right]=P_{q q}^{(0)}(x)-C_{\mathrm{F}}(1-x) .
\end{aligned}
$$

Note that the first moments of both $P_{q q}^{(0)}$ and $\Delta P_{q q}^{(0)}$ vanish (leading to conservation laws and sum rules), but not so of $\Delta_{T} P_{q q}^{(0)}$. The effects of evolution are shown in Fig. 3 .
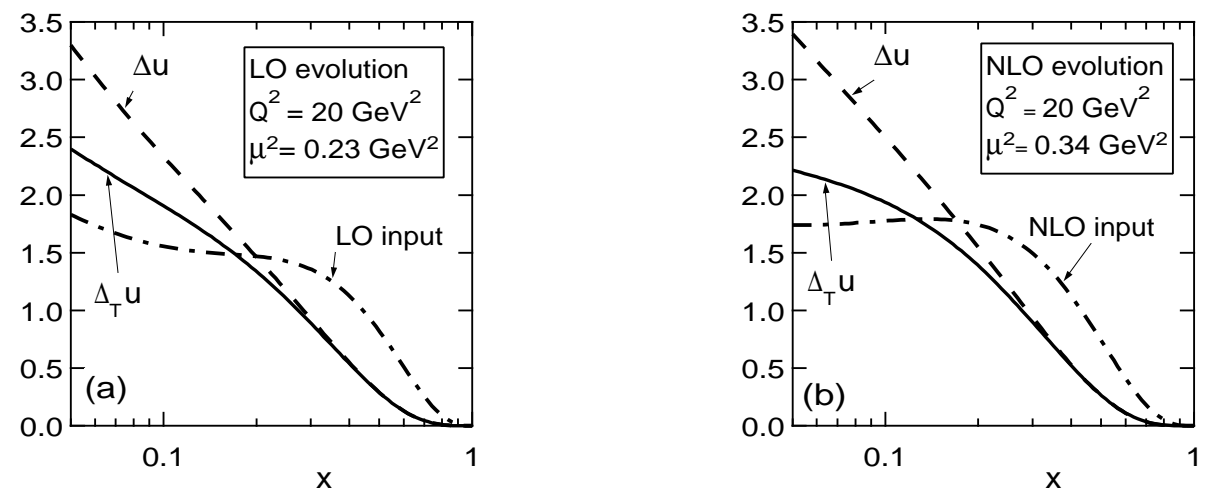

FIGURE 3. The $Q^{2}$-evolution of $\Delta_{T} u\left(x, Q^{2}\right)$ and $\Delta u\left(x, Q^{2}\right)$ compared at (a) LO and (b) NLO; from [19].

By considering hadron-parton helicity amplitudes (see the figure alongside), Soffer [20] constructed an interesting bound involving transversity. Taking into account all relevant symmetries there are only two independent amplitudes, in terms of which all three densities are expressed:

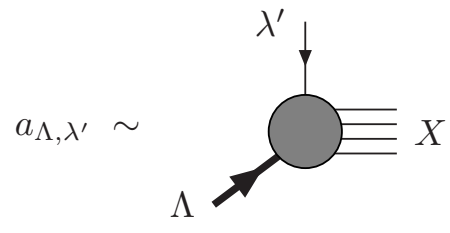

$$
\begin{aligned}
f(x) & \propto \operatorname{Im}\left(A_{++,++}+A_{+-,+-}\right) & \propto \sum_{X}\left(a_{++}^{*} a_{++}+a_{+-}^{*} a_{+-}\right), \\
\Delta f(x) & \propto \operatorname{Im}\left(A_{++,++}-A_{+-,+-}\right) & \propto \sum_{X}\left(a_{++}^{*} a_{++}-a_{+-}^{*} a_{+-}\right), \\
\Delta_{T} f(x) & \propto \operatorname{Im} A_{+-,-+} & \propto \sum_{X} a_{--}^{*} a_{++}
\end{aligned}
$$


A straight-forward Schwartz-type inequality: $\sum_{X}\left|a_{++} \pm a_{--}\right|^{2} \geq 0$ then translates into $f_{+}(x) \geq\left|\Delta_{T} f(x)\right|$ or $f(x)+\Delta f(x) \geq 2\left|\Delta_{T} f(x)\right|$, which is precisely the Soffer bound. Notice that it involves all three leading-twist structures.

\section{A DIS DEFINITION FOR TRANSVERSITY}

The other twist-two densities are naturally defined via DIS, where the parton picture is formulated and many model calculations performed. When translated to Drell-Yan (DY) processes, large $K$ factors appear $\sim \mathrm{O}\left(\pi \alpha_{s}\right)$. At RHIC energies this corresponds to $\mathrm{a} \sim 30 \%$ correction, at EMC/SMC nearly $100 \%$. Pure DY coefficient functions are known, but are scheme dependent. Moreover, a $\ln ^{2} x /(1-x)$ term appears that is not found for spin-averaged or helicity-dependent DY. Together with recent problems arising in connection with vector-scalar current products, this suggests an interesting check.

One might invoke a Higgs-photon interference mechanism, which, though experimentally hardly viable, does provide a DIS-type definition for transversity since the presence of a scalar vertex forces chirality flip. Care must be taken over the extra renormalisation contribution from the scalar vertex,

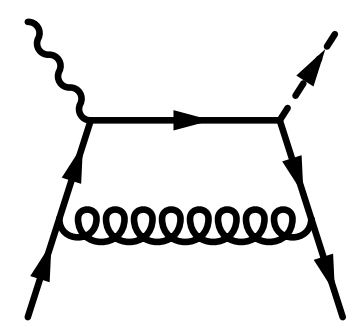

FIGURE 4. Hypothetical Higgs-photon interference mechanism. which factorises into the running mass (or Higgs coupling). One then needs to calculate diagrams of the form of Fig. 4 (and correspondents for DY) in order to obtain the relevant higher-order Wilson coefficients.

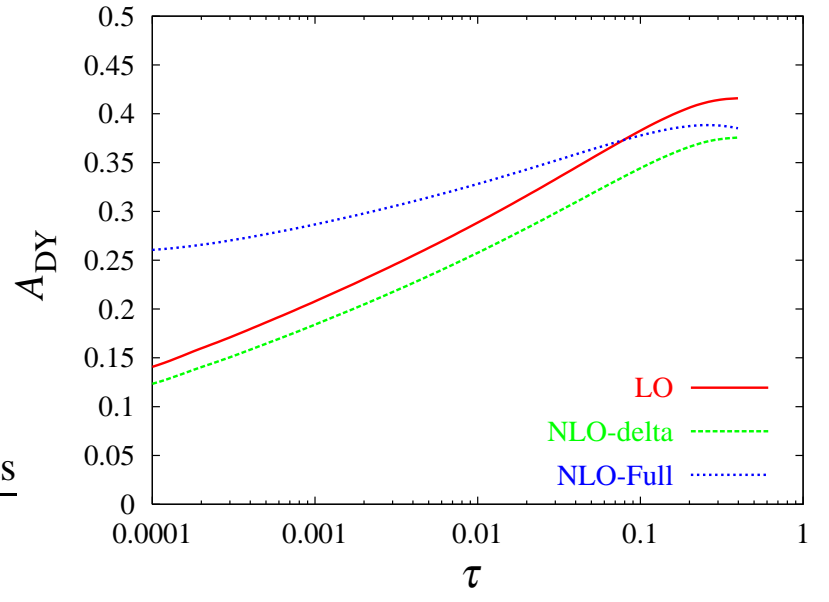

FIGURE 5. Transversity asymmetry (valence only) for DY; $\tau=Q^{2} / s, s=4 \cdot 10^{4} \mathrm{GeV}^{2}$.

Fig. 5 illustrates the effect of the next-to-leading order (NLO) Wilson coefficient [21]. In contrast to the helicity asymmetry [22], where the difference between the LO and NLO is small (the large coefficient of the $\delta$-function is identical in the numerator and denominator), here there are important differences between the spin-averaged denominator and the transversity-dependent numerator. The principal culprits are the $\delta$ function coefficient and the new $\frac{\ln ^{2} x}{1-x}$ term. The DIS-DY "transformation" coefficients for the unpolarised and transversity cases are:

$$
\begin{array}{r}
C_{q, D Y}^{f}-2 C_{q, D I S}^{f}=\frac{\alpha_{s}}{2 \pi} \frac{4}{3}\left[\frac{3}{(1-z)_{+}}+2\left(1+z^{2}\right)\left(\frac{\ln (1-z)}{1-z}\right)_{+}-6-4 z\right. \\
\left.+\left(\frac{4}{3} \pi^{2}+1\right) \delta(1-z)\right]
\end{array}
$$




$$
\begin{array}{r}
C_{q, D Y}^{h}-2 C_{q, D I S}^{h}=\frac{\alpha_{S}}{2 \pi} \frac{4}{3}\left[\frac{3 z}{(1-z)_{+}}+4 z\left(\frac{\ln (1-z)}{1-z}\right)_{+}-6 z \frac{\ln ^{2} z}{1-z}+4(1-z)\right. \\
\left.+\left(\frac{4}{3} \pi^{2}-1\right) \delta(1-z)\right]
\end{array}
$$

\section{T-ODD STRUCTURES}

We now wish to generalise the $\mathbf{k}_{\perp}$-integrated density functions to include all possible correlations between the quark and parent-hadron spins, later on we shall find we also need $\mathbf{k}_{\perp}$-dependent generalisations. Thus, some extra notation will be required (see [13]). In this way objects like $\Delta_{L}^{T} f$ have a simple interpretation:

- subscripts $0, L$ and $T$ in density and fragmentation functions denote the quark polarisation state,

- superscripts $0, L$ and $T$ denote the parent or off-spring hadron polarisation state.

The superscript is dropped when equal to the subscript.

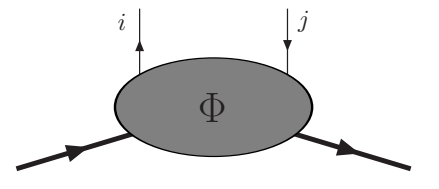

FIGURE 6. Quark-quark correlation matrix.

The aim then is to parametrise the quark-quark correlation matrix (see Fig. 6) in the most general manner, while respecting the natural properties of hermiticity, parity, and time-reversal invariance, though, as we shall see later, this last may be relaxed. The most general decomposition of $\Phi$ over a complete basis of Dirac matrices is

$$
\Phi(k, P, S)=\frac{1}{2}\left\{S \mathbb{I}+V_{\mu} \gamma^{\mu}+A_{\mu} \gamma_{5} \gamma^{\mu}+\mathrm{i} P_{5} \gamma_{5}+\mathrm{i} T_{\mu \nu} \sigma^{\mu v} \gamma_{5}\right\},
$$

where the quantities $S, V^{\mu}, A^{\mu}, P_{5}$ and $T^{\mu v}$ are to be constructed from the vectors $k^{\mu}$, $P^{\mu}$ and the pseudovector $S^{\mu}$.

Relaxing $T$ invariance allows two new twist-two structures:

$$
V^{\mu}=\cdots+\frac{1}{M} A_{1}^{\prime} \varepsilon^{\mu v \rho \sigma} P_{v} k_{\perp \rho} S_{\perp \sigma} \quad \text { and } \quad T^{\mu \nu}=\cdots+\frac{1}{M} A_{2}^{\prime} \varepsilon^{\mu v \rho \sigma} P_{\rho} k_{\perp \sigma} .
$$

These give rise to two $\mathbf{k}_{\perp}$-dependent $T$-odd density functions, $f_{1 T}^{\perp}$ and $h_{1}^{\perp}$ [23]:

$$
\Phi^{\left[\gamma^{+}\right]}=\cdots-\frac{\varepsilon_{\perp}^{i j} k_{\perp i} S_{\perp j}}{M} f_{1 T}^{\perp}\left(x, \mathbf{k}_{\perp}^{2}\right) \quad \text { and } \quad \Phi^{\left[\mathrm{i} \sigma^{i+} \gamma_{5}\right]}=\cdots-\frac{\varepsilon_{\perp}^{i j} k_{\perp j}}{M} h_{1}^{\perp}\left(x, \mathbf{k}_{\perp}^{2}\right) .
$$

The partonic interpretation is as follows. The density $f_{1 T}^{\perp}$ relates to the number density of unpolarised quarks in a transversely polarised nucleon:

$$
\begin{aligned}
P_{q / N \uparrow}\left(x, \mathbf{k}_{\perp}\right)-P_{q / N \downarrow}\left(x, \mathbf{k}_{\perp}\right) & =P_{q / N \uparrow}\left(x, \mathbf{k}_{\perp}\right)-P_{q / N \uparrow}\left(x,-\mathbf{k}_{\perp}\right) \\
& =-2 \frac{\left|\mathbf{k}_{\perp}\right|}{M} \sin \left(\phi_{k}-\phi_{S}\right) f_{1 T}^{\perp}\left(x, \mathbf{k}_{\perp}^{2}\right) .
\end{aligned}
$$

The $T$-odd density $h_{1}^{\perp}$ measures quark transverse polarisation in an unpolarised hadron:

$$
P_{q \uparrow / N}\left(x, \mathbf{k}_{\perp}\right)-P_{q \downarrow / N}\left(x, \mathbf{k}_{\perp}\right)=-\frac{\left|\mathbf{k}_{\perp}\right|}{M} \sin \left(\phi_{k}-\phi_{S}\right) h_{1}^{\perp}\left(x, \mathbf{k}_{\perp}^{2}\right) .
$$


It is convenient to define two quantities $\Delta_{0}^{T} f$ and $\Delta_{T}^{0} f$ (related to $f_{1 T}^{\perp}$ and $h_{1}^{\perp}$ respectively) by absorbing the factors $\left|\mathbf{k}_{\perp}\right| / M$ :

$$
\Delta_{0}^{T} f\left(x, \mathbf{k}_{\perp}^{2}\right) \equiv-2 \frac{\left|\mathbf{k}_{\perp}\right|}{M} f_{1 T}^{\perp}\left(x, \mathbf{k}_{\perp}^{2}\right) \quad \text { and } \quad \Delta_{T}^{0} f\left(x, \mathbf{k}_{\perp}^{2}\right) \equiv-\frac{\left|\mathbf{k}_{\perp}\right|}{M} h_{1}^{\perp}\left(x, \mathbf{k}_{\perp}^{2}\right) .
$$

The question now arises as to why we should entertain such $T$-odd quantities at all. There are various attitudes: Anselmino et al. [24] (among others) advocate initial-state effects, which prevent implementation of naïve time-reversal invariance. The suggestion is that the colliding hadrons interact strongly with non-trivial relative phases, akin to those arising from final-state effects. An alternative has been proposed by Anselmino et al. [25]: they apply a general argument on time reversal for particle multiplets suggested by Weinberg [26]. If the internal structure of hadrons is described at some low momentum scale by a chiral lagrangian, time reversal might be realised in a "non-standard" manner that could mix the multiplet components. According to this approach, the $u(d)$ density transforms into the $d(u)$ density, and time-reversal invariance simply establishes a relation between the $u$ and $d$ sectors.

Finally, Collins [27] has recently reconsidered his proof of the vanishing of $f_{1 T}^{\perp}$ and $h_{1}^{\perp}$, based on the field-theoretical expressions of the two densities. He noticed that on reinstating the link operators into quark-quark bi-locals the densities do not simply change sign under $T$; a future-pointing Wilson line becomes past-pointing. Consequently, time-reversal invariance, does not constrain $f_{1 T}^{\perp}$ and $h_{1}^{\perp}$ to be zero, but relates processes probing Wilson lines in opposite directions. Collins thus predicts the Sivers asymmetry, e.g., to have opposite signs in DIS and in DY.

\section{LEPTON-NUCLEON SCATTERING}

One might hope to access transversity through exclusive leptoproduction of vector mesons (see Fig. 7) alongside). However, Mankiewicz et al. [28] showed that the chirallyodd contribution to vector-meson production is actually zero at LO in $\alpha_{s}$. Diehl et al. [29] and Collins et al. [30] later extended this, observing that the chirally-odd contribution vanishes due to angular-momentum and chirality conservation in the hard scattering and so holds at leading twist to all orders in $\alpha_{s}$. Thus, exclusive vector-meson leptoproduction cannot probe (off-diagonal) transversity densities.

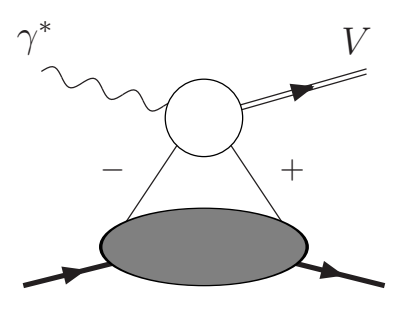

The cross-section for production off a longitudinally polarised target is [31]:

$$
\begin{aligned}
\frac{\mathrm{d}^{5} \sigma\left(\lambda_{N}\right)}{\mathrm{d} x \mathrm{~d} y \mathrm{~d} z \mathrm{~d}^{2} \mathbf{P}_{h \perp}}=- & \frac{4 \pi \alpha_{\mathrm{em}}^{2} s}{Q^{4}} \lambda_{N} \sum_{a} e_{a}^{2} x(1-y) \sin \left(2 \phi_{h}\right) \\
& \times I\left[\frac{2\left(\hat{\mathbf{h}} \cdot \kappa_{\perp}\right)\left(\hat{\mathbf{h}} \cdot \mathbf{k}_{\perp}\right)-\kappa_{\perp} \cdot \mathbf{k}_{\perp}}{M M_{h}} h_{1 L a}^{\perp}\left(x, \mathbf{k}_{\perp}\right) H_{1 a}^{\perp}\left(z, \kappa_{\perp}\right)\right] .
\end{aligned}
$$

Transversity is not present here, but the asymmetry does depend on the Collins function $H_{1}^{\perp} \propto \sin \left(2 \phi_{h}\right)$, also on a $\mathbf{k}_{\perp}$-dependent density function $h_{1 L}^{\perp}$. 
Summarising, in the context of semi-inclusive DIS there are four candidate leadingtwist reactions to determine $\Delta_{T} f$ : namely, inclusive leptoproduction of

1. a transversely polarised hadron from a transversely polarised target;

2. an unpolarised hadron from a transversely polarised target;

3. two hadrons from a transversely polarised target;

4. a spin-one polarised or unpolarised hadron from a transversely polarised target.

\section{HADRON-HADRON SCATTERING}

Let us now examine single-hadron production with a transversely polarised target. The process is exemplified in Fig. 8 alongside: $A$ is transversely polarised and the unpolarised (or spinless) hadron $h$ is produced at large transverse momentum $\mathbf{P}_{h T}$, therefore $\mathrm{pQCD}$ is applicable. In typical experiments $A$ and $B$ are protons while $h$ is a pion. According to the factorisation theorem, the differential cross-section for the reaction may be written formally as

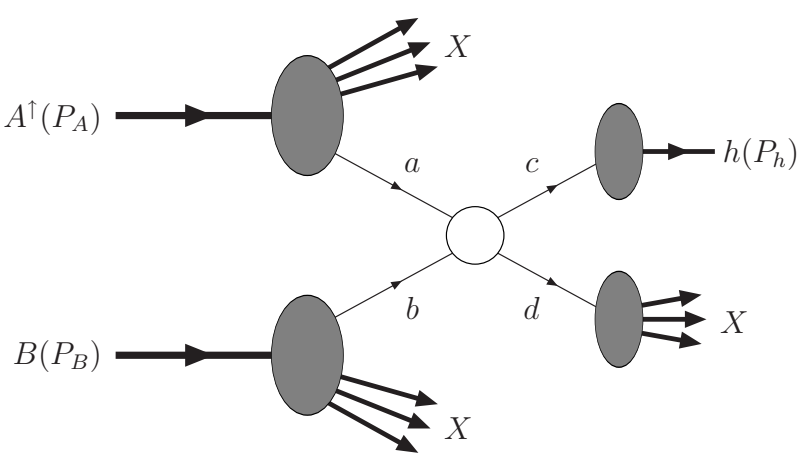

FIGURE 8. Single-hadron production with a transversely polarised target.

$$
\mathrm{d} \sigma=\sum_{a b c} \sum_{\alpha \alpha^{\prime} \gamma \gamma^{\prime}} \rho_{\alpha^{\prime} \alpha}^{a} f_{a}\left(x_{a}\right) \otimes f_{b}\left(x_{b}\right) \otimes \mathrm{d} \hat{\sigma}_{\alpha \alpha^{\prime} \gamma \gamma^{\prime}} \otimes D_{h / c}^{\gamma^{\prime} \gamma}(z)
$$

$f_{a}\left(f_{b}\right)$ is the density of parton $a(b)$ inside hadron $A(B), \rho_{\alpha \alpha^{\prime}}^{a}$ is the parton $a$ spin density matrix, $D_{h / c}^{\gamma \gamma^{\prime}}$ is the fragmentation matrix of parton $c$ into hadron $h$ and $\mathrm{d} \hat{\sigma} / \mathrm{d} \hat{t}$ is the elementary cross-section:

$$
\left(\frac{\mathrm{d} \hat{\sigma}}{\mathrm{d} \hat{t}}\right)_{\alpha \alpha^{\prime} \gamma \gamma^{\prime}}=\frac{1}{16 \pi \hat{s}^{2}} \frac{1}{2} \sum_{\beta \delta} M_{\alpha \beta \gamma \delta} M_{\alpha^{\prime} \beta \gamma^{\prime} \delta}^{*} .
$$

Here $M_{\alpha \beta \gamma \delta}$ is the amplitude for the hard partonic process, displayed in Fig. 9. For an unpolarised produced hadron, the off-diagonal elements of $D_{h / c}^{\gamma \gamma^{\prime}}$ vanish, i.e., $D_{h / c}^{\gamma \gamma^{\prime}} \propto \delta_{\gamma \gamma^{\prime}}$. Helicity conservation then implies $\alpha=\alpha^{\prime}$ and thus there can be no dependence on the spin of hadron $A$. Consequently, all SSA's must vanish.

To avoid this conclusion, intrinsic quark transverse motion or higher-twist effects must be invoked; this can be

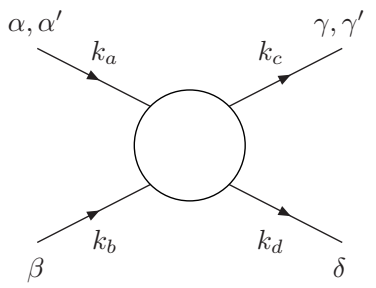

FIGURE 9. Partonic hard scattering amplitude. done in three different ways:

1. $\kappa_{T}$ in hadron $h$ allows $D_{h / c}^{\gamma \gamma^{\prime}}$ to be non-diagonal (a fragmentation $T$-odd effect), the Collins effect [32]; 
2. $\mathbf{k}_{T}$ in hadron $A$ implies that $f_{a}\left(x_{a}\right)$ should be replaced by the $P_{a}\left(x_{a}, \mathbf{k}_{T}\right)$, which may depend on the spin of hadron $A$ (a density $T$-odd effect), the Sivers effect [33];

3. $\mathbf{k}_{T}^{\prime}$ in hadron $B$ implies that $f_{b}\left(x_{b}\right)$ should be replaced by $P_{b}\left(x_{b}, \mathbf{k}_{T}^{\prime}\right)$; a transverse spin of parton $b$ in the unpolarised hadron $B$ may then couple to the transverse spin of parton $a$ in $A$ (a density $T$-odd effect), see [34].

It should be stressed that all these intrinsic- $\kappa_{T},-\mathbf{k}_{T}$, or $-\mathbf{k}_{T}^{\prime}$ effects are $T$-odd. Note too that when intrinsic quark transverse motion is taken into account, the QCD factorisation theorem is not proven.

Assuming, for discussion purposes, factorisation to be valid, the cross-section is

$$
\begin{aligned}
E_{h} \frac{\mathrm{d}^{3} \sigma}{\mathrm{d}^{3} \mathbf{P}_{h}}= & \sum_{a b c} \sum_{\alpha \alpha^{\prime} \beta \beta^{\prime} \gamma \gamma^{\prime}} \int \mathrm{d} x_{a} \int \mathrm{d} x_{b} \int \mathrm{d}^{2} \mathbf{k}_{T} \int \mathrm{d}^{2} \mathbf{k}_{T}^{\prime} \int \mathrm{d}^{2} \kappa_{T} \frac{1}{\pi z} \\
& \times P_{a}\left(x_{a}, \mathbf{k}_{T}\right) \rho_{\alpha^{\prime} \alpha}^{a} P_{b}\left(x_{b}, \mathbf{k}_{T}^{\prime}\right) \rho_{\beta^{\prime} \beta}^{b}\left(\frac{\mathrm{d} \hat{\sigma}}{\mathrm{d} \hat{t}}\right)_{\alpha \alpha^{\prime} \beta \beta^{\prime} \gamma \gamma^{\prime}} D_{h / c}^{\gamma^{\prime} \gamma}\left(z, \kappa_{T}\right),
\end{aligned}
$$

where

$$
\left(\frac{\mathrm{d} \hat{\sigma}}{\mathrm{d} \hat{t}}\right)_{\alpha \alpha^{\prime} \beta \beta^{\prime} \gamma \gamma^{\prime}}=\frac{1}{16 \pi \hat{s}^{2}} \sum_{\delta} M_{\alpha \beta \gamma \delta} M_{\alpha^{\prime} \beta^{\prime} \gamma^{\prime} \delta}^{*} .
$$

The Collins mechanism requires intrinsic quark transverse motion inside the produced hadron $h$ while neglecting all other quark transverse momenta (the spin of $A$ is along $y$ ):

$$
\begin{aligned}
E_{h} \frac{\mathrm{d}^{3} \sigma\left(\mathbf{S}_{T}\right)}{\mathrm{d}^{3} \mathbf{P}_{h}}-E_{h} \frac{\mathrm{d}^{3} \sigma\left(-\mathbf{S}_{T}\right)}{\mathrm{d}^{3} \mathbf{P}_{h}}=-2\left|\mathbf{S}_{T}\right| \sum_{a b c} \int \mathrm{d} x_{a} \int \frac{\mathrm{d} x_{b}}{\pi z} \int \mathrm{d}^{2} \kappa_{T} \\
\quad \times \Delta_{T} f_{a}\left(x_{a}\right) f_{b}\left(x_{b}\right) \Delta_{T T} \hat{\sigma}\left(x_{a}, x_{b}, \kappa_{T}\right) \Delta_{T}^{0} D_{h / c}\left(z, \kappa_{T}^{2}\right),
\end{aligned}
$$

where $\Delta_{T T} \hat{\sigma}$ is a partonic spin-transfer asymmetry. The Sivers effect relies on $T$-odd density functions and predicts a form

$$
\begin{aligned}
& E_{h} \frac{\mathrm{d}^{3} \boldsymbol{\sigma}\left(\mathbf{S}_{T}\right)}{\mathrm{d}^{3} \mathbf{P}_{h}}-E_{h} \frac{\mathrm{d}^{3} \sigma\left(-\mathbf{S}_{T}\right)}{\mathrm{d}^{3} \mathbf{P}_{h}}=\left|\mathbf{S}_{T}\right| \sum_{a b c} \int \mathrm{d} x_{a} \int \frac{\mathrm{d} x_{b}}{\pi z} \int \mathrm{d}^{2} \mathbf{k}_{T} \\
& \times \Delta_{0}^{T} f_{a}\left(x_{a}, \mathbf{k}_{T}^{2}\right) f_{b}\left(x_{b}\right) \frac{\mathrm{d} \hat{\sigma}\left(x_{a}, x_{b}, \mathbf{k}_{T}\right)}{\mathrm{d} \hat{t}} D_{h / c}(z),
\end{aligned}
$$

where $\Delta_{0}^{T} f$ (related to $f_{1 T}^{\perp}$ ) is a $T$-odd density. Finally, the effect studied by Boer gives rise to an asymmetry involving the other $T$-odd density $\Delta_{T}^{0} f$ (related to $h_{1}^{\perp}$ ):

$$
\begin{aligned}
E_{h} \frac{\mathrm{d}^{3} \sigma\left(\mathbf{S}_{T}\right)}{\mathrm{d}^{3} \mathbf{P}_{h}}-E_{h} \frac{\mathrm{d}^{3} \sigma\left(-\mathbf{S}_{T}\right)}{\mathrm{d}^{3} \mathbf{P}_{h}}=-2\left|\mathbf{S}_{T}\right| \sum_{a b c} \int \mathrm{d} x_{a} \int \frac{\mathrm{d} x_{b}}{\pi z} \int \mathrm{d}^{2} \mathbf{k}_{T}^{\prime} \\
\quad \times \Delta_{T} f_{a}\left(x_{a}\right) \Delta_{T}^{0} f_{b}\left(x_{b}, \mathbf{k}_{T}^{\prime 2}\right) \Delta_{T T} \hat{\sigma}^{\prime}\left(x_{a}, x_{b}, \mathbf{k}_{T}^{\prime}\right) D_{h / c}(z),
\end{aligned}
$$

where $\Delta_{T T} \hat{\sigma}^{\prime}$ is a partonic initial-spin correlation.

As already mentioned, Efremov et al. [35] pointed out that SSA's can arise in pQCD at higher twist via gluonic poles in diagrams involving $q q g$ correlators. Such asymmetries 
were evaluated by Qiu and Sterman, who studied direct photon production [17, 18] and hadron production [36]. The extension to chirally-odd contributions was made by Kanazawa et al. [37, 38]. The results may be summarised in

$$
\begin{aligned}
& \mathrm{d} \sigma=\sum_{a b c}\left\{G_{F}^{a}\left(x_{a}, y_{a}\right) \otimes f_{b}\left(x_{b}\right) \otimes \mathrm{d} \hat{\sigma} \otimes D_{h / c}(z)\right. \\
&+\Delta_{T} f_{a}\left(x_{a}\right) \otimes E_{F}^{b}\left(x_{b}, y_{b}\right) \otimes \mathrm{d} \hat{\sigma}^{\prime} \otimes D_{h / c}(z) \\
&\left.\quad+\Delta_{T} f_{a}\left(x_{a}\right) \otimes f_{b}\left(x_{b}\right) \otimes \mathrm{d} \hat{\sigma}^{\prime \prime} \otimes D_{h / c}^{(3)}(z)\right\} .
\end{aligned}
$$

The first term (not containing transversity) is a chirally-even mechanism studied by Qiu and Sterman, the second term is the chirally-odd contribution analysed by Kanazawa and Koike, and the third contains a twist-three fragmentation function $D_{h / c}^{(J)} \cdot$

Admitting twist-three contributions, the SSA in DY is [39]

$$
\begin{aligned}
A_{T}^{\mathrm{DY}}=\left|\mathbf{S}_{1 \perp}\right| \frac{2 \sin 2 \theta}{1+\cos ^{2} \theta} \sin \left(\phi-\phi_{S_{1}}\right) \frac{M}{Q} \\
\times \frac{\sum_{a} e_{a}^{2}\left[x_{1} \widetilde{f}_{T}^{a}\left(x_{1}\right) \bar{f}_{a}\left(x_{2}\right)+x_{2} \Delta_{T} f_{a}\left(x_{1}\right) \bar{h}_{a}\left(x_{2}\right)\right]}{\sum_{a} e_{a}^{2} f_{a}\left(x_{1}\right) \bar{f}_{a}\left(x_{2}\right)},
\end{aligned}
$$

where $\tilde{f}_{T}(x)$ and $\bar{h}(x)$ are twist-three $T$-odd density functions. The existence of such $T$-odd density functions has been advocated by Boer [34] to explain an anomalously large $\cos 2 \phi$ term seen in unpolarised DY data. As presented, such contributions would require initial-state interactions - this may be considered unlikely. Hammon et al. [40] have shown that SSA's may arise from gluonic poles in twist-three multiparton correlation functions (see Fig. 10 alongside). The corresponding SSA is then

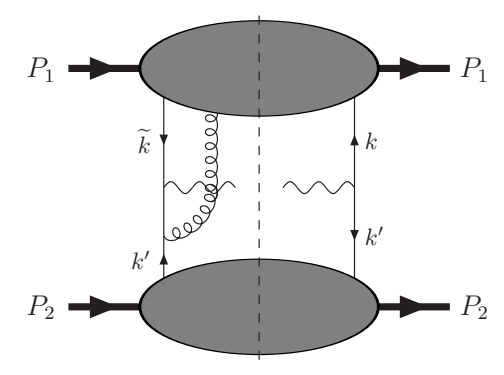

FIGURE 10. A twist-three gluonpole contribution to DY.

$$
\begin{aligned}
& A_{T}^{\mathrm{DY}} \propto\left|\mathbf{S}_{1 \perp}\right| \frac{2 \sin 2 \theta}{1+\cos ^{2} \theta} \sin \left(\phi-\phi_{S_{1}}\right) \frac{M}{Q} \\
& \times \frac{\sum_{a} e_{a}^{2}\left[G_{F}^{a}\left(x_{1}, x_{1}\right) \bar{f}_{a}\left(x_{2}\right)+\Delta_{T} f_{a}\left(x_{1}\right) E_{F}^{a}\left(x_{2}, x_{2}\right)\right]}{\sum_{a} e_{a}^{2} f_{a}\left(x_{1}\right) \bar{f}_{a}\left(x_{2}\right)} .
\end{aligned}
$$

Comparing this with the previous expression we may identify

$$
\begin{aligned}
f_{T}^{\mathrm{eff}}(x) & \sim G_{F}(x, x) \sim \int \mathrm{d} y \operatorname{Im} G_{A}^{\mathrm{eff}}(x, y), \\
h^{\mathrm{eff}}(x) & \sim E_{F}(x, x) \sim \int \mathrm{d} y \operatorname{Im} E_{A}^{\mathrm{eff}}(x, y) .
\end{aligned}
$$

Thus, $T$-odd functions at twist three, can explain $A_{T}^{\mathrm{DY}}$ via quark-gluon interactions, without initial-state effects. 


\section{CONCLUSIONS}

The study of single-spin asymmetries has become a very complex area of high-energy spin physics. A plethora of new structure and fragmentation functions has opened the way to explaining much existing phenomenology. However, in order to distinguish and separate out the various mechanisms proposed, a large amount of diverse high-energy data will be necessary and it is difficult (if not indeed irrelevant and even misleading) to single out at a few key experiments. In other words, all new data will be very welcome.

\section{REFERENCES}

1. B. Andersson, G. Gustafson and G. Ingelman, Phys. Lett. B85 (1979) 417.

2. T.A. DeGrand and H.I. Miettinen, Phys. Rev. D24 (1981) 2419; erratum, ibid. D31 (1985) 661.

3. R. Barni, G. Preparata and P.G. Ratcliffe, Phys. Lett. B296 (1992) 251.

4. J. Soffer and N.A. Tornqvist, Phys. Rev. Lett. 68 (1992) 907.

5. J. Ralston and D.E. Soper, Nucl. Phys. B152 (1979) 109.

6. F. Baldracchini, N.S. Craigie, V. Roberto and M. Socolovsky, Fortschr. Phys. 30 (1981) 505.

7. X. Artru and M. Mekhfi, Z. Phys. C45 (1990) 669.

8. J. Kodaira, S. Matsuda, K. Sasaki and T. Uematsu, Nucl. Phys. B159 (1979) 99.

9. I. Antoniadis and C. Kounnas, Phys. Rev. D24 (1981) 505.

10. A.P. Bukhvostov, É.A. Kuraev, L.N. Lipatov and G.V. Frolov, Nucl. Phys. B258 (1985) 601.

11. P.G. Ratcliffe, Nucl. Phys. B264 (1986) 493.

12. R.L. Jaffe and X.-D. Ji, Nucl. Phys. B375 (1992) 527.

13. V. Barone, A. Drago and P.G. Ratcliffe, Phys. Rep. 359 (2002) 1; hep-ph/0104283.

14. V. Barone and P.G. Ratcliffe, Transverse Polarisation of Quarks in Hadrons (World Sci., in press).

15. G.L. Kane, J. Pumplin and W. Repko, Phys. Rev. Lett. 41 (1978) 1689.

16. A.V. Efremov and O.V. Teryaev, Phys. Lett. B150 (1985) 383.

17. J.-W. Qiu and G. Sterman, Phys. Rev. Lett. 67 (1991) 2264.

18. J.-W. Qiu and G. Sterman, Nucl. Phys. B378 (1992) 52.

19. A. Hayashigaki, Y. Kanazawa and Y. Koike, Phys. Rev. D56 (1997) 7350; hep-ph/9707208.

20. J. Soffer, Phys. Rev. Lett. 74 (1995) 1292; hep-ph/9409254.

21. P.G. Ratcliffe, work in progress.

22. P.G. Ratcliffe, Nucl. Phys. B223 (1983) 45.

23. D. Boer and P.J. Mulders, Phys. Rev. D57 (1998) 5780; hep-ph/9711485.

24. M. Anselmino and F. Murgia, Phys. Lett. B442 (1998) 470; hep-ph/9808426.

25. M. Anselmino, V. Barone, A. Drago and F. Murgia, Non-standard time reversal and transverse singlespin asymmetries, hep-ph/0209073.

26. S. Weinberg, The Quantum Theory of Fields. Vol. 1: Foundations (Cambridge U. Press, 1995).

27. J.C. Collins, Phys. Lett. B536 (2002) 43; hep-ph/0204004.

28. L. Mankiewicz, G. Piller and T. Weigl, Eur. Phys. J. C5 (1998) 119; hep-ph/9711227.

29. M. Diehl, T. Gousset and B. Pire, Phys. Rev. D59 (1999) 034023; hep-ph/9808479.

30. J.C. Collins and M. Diehl, Phys. Rev. D61 (2000) 114015; hep-ph/9907498.

31. A.M. Kotzinian and P.J. Mulders, Phys. Lett. B406 (1997) 373; hep-ph/9701330.

32. J.C. Collins, Nucl. Phys. B396 (1993) 161; hep-ph/9208213.

33. D. Sivers, Phys. Rev. D41 (1990) 83.

34. D. Boer, Phys. Rev. D60 (1999) 014012; hep-ph/9902255.

35. A.V. Efremov and O.V. Teryaev, Yad. Fiz. 36 (1982) 242; transl., Sov. J. Nucl. Phys. 36 (1982) 140.

36. J.-W. Qiu and G. Sterman, Phys. Rev. D59 (1999) 014004; hep-ph/9806356.

37. Y. Kanazawa and Y. Koike, Phys. Lett. B478 (2000) 121; hep-ph/0001021

38. Y. Kanazawa and Y. Koike, Phys. Lett. B490 (2000) 99; hep-ph/0007272.

39. D. Boer, P.J. Mulders and O.V. Teryaev, Phys. Rev. D57 (1997) 3057; hep-ph/9710223.

40. N. Hammon, O. Teryaev and A. Schäfer, Phys. Lett. B390 (1997) 409; hep-ph/9611359. 